\title{
Reviewer Responses
}

Thank you for the excellent reviews; we believe that having addressed them has improved the quality of the paper. We provide a detailed response to each of the reviewers' points below, and also provide a revised manuscript based on the reviewers' questions and suggestions.

Reviewer \#1: The paper presents a new Matrix Factorization Model for widefield calcium imaging data. The main contributions of this new model are:

- The Temporal components $\mathrm{C}$ are not constrained to be non-negative.

- The spatial components are initialized taking into account information from Allen CCF Brain Atlas. This information is also taken into account in the model, so as to encourage the spatial components to be coherent with them.

- The authors provide public CPU and GPU implementations of their methods in a complete suite for treating the entire widefield calcium process.

On the positive side, their approach incorporates knowledge (through the use of brain atlas) in the process and guides the solution towards biological meaning and stablity, through different runs. This can, indeed, be seen as a contribution to the most challenging shortcoming of Matrix Factorization methods: Their high dependence on the initialization of the components. Still, this doesn't fix the fact that the modeled computational problem is not solvable in polynomial time, which means the algorithms for solving the model may only be heuristic. Hence, the effectiveness of the technique can only be established through specific experiments. Indeed, the authors present interesting experiments that support their idea of constraining the location of the neurons to specific brain regions, which may help eliminate false positives.

We thank the reviewer for the comments and agree with them.

The main drawback of the paper is in the comparison with only SVD. This is insufficient to support the experimental value of the proposed technique. Although the type of experiments performed are interesting and support the value of the work, I don't think that SVD methods, with which they compare, can be considered state-of-the-art. I think the paper has strong points, but should compare with techniques such as CNMF, Suite2P and HNCCorr. Furthermore, it would be interesting to see how the proposed method performs on the Neurofinder Dataset. The comparison with SVD was chosen because it is the current state-of-the-art for Widefield Calcium Imaging (WFCl) data that lacks cellular resolution, and has been used in multiple papers, for example, most recently in Musall et al., Nature Neuroscience, 2019 and Shimaoka et al., ELife, 2019. Indeed, no other decomposition methods have been used on this kind of data, that the authors are aware of. We do compare our findings to just using semi-Nonnegative Matrix Factorization without the localization constraint, as presented in the supplementary materials. The methods suggested by the reviewer (CNMF, Suite2P and HNCCorr) are only applicable to data with cellular resolution, which WFCl does not support; see the Introduction for further discussion of this point. 
Reviewer \#2: The main finding of this study is to propose a novel algorithm named 'LocalNMF' that decomposes widefield $\mathrm{Ca}$ imaging (WFCl) data into spatial and temporal components. The LocalNMF uses a brain atlas to initialize the estimated spatial components such that the spread of the spatial component is limited and localized in the different brain regions. The resulting components lead to a more interpretable decomposition of WFCl data. Its efficiency was validated with multiple experimental datasets related to binary behavioural features and continuous behavioural variables.

The authors did a superb job in designing the study and the experiments were conducted well. The data clearly support the conclusions of this study. The presentation of the results is good and the discussion is clear. Overall, this is a very interesting study that puts forward clear evidence for using NMF for analysing data revealed in neuroscience. I would like to recommend it for publication. However, the following comments may help to improve the writing and readability of the current version of the manuscript.

Thank you for the comments!

(1) Line 64: The main advancement of this study is stated as "a new approach to perform a localized, more interpretable decomposition of WFCl data. The proposed approach is a variation on classical NMF, termed localized semi-NMF (LocaNMF), that decomposes the widefield activity by (a) using existing brain atlases to initialize the estimated spatial components, and (b) limiting the spread of each spatial component in order to obtain localized components." Thus the authors emphasize that both (a) and (b) are important. If I understand it correctly, these points are related to the discussion on Sec. Comparison with vanilla NMF on line 200. My question here is about the results by using vanilla NMF, e.g., four Suppl. figures. Initially, I thought that these results are obtained by the vanilla NMF with the SAME brain atlas initialization. However, in the end, the Methods part at line 576, I found that "We use vanilla NMF with random initialization as a comparison to LocaNMF". Thus for comparison, there are some incomplete issues. For vanilla NMF, it can capture similar behaviors as in LocaNMF. Indeed, since the vanilla NMF or NMF, in general, is a method for detecting the localized feature. As shown in all of four SI figures, NMF can capture some similar behaviors as in locaNMF. For example, as in Fig SI 4, SSp : L barrel field is comparable for both NMF and locaNMF. Therefore, the localNMF employs a localization constraint (Eq. 5) to improve the robustness of the NMF. This is an advantage to overcome the non-robustness. I wonder if other tricks can also play the same role. There are many tricks to get rid of the local minimums during the convergence of NMF. Certainly, the current one, locaNMF, may be the most efficient and best one in this application scenario. However, to demonstrate this, one snapshot of the results is not enough, i.e., the results of four SI figures are outcomes of one single run of vanilla NMF. For the same dataset, suppose there are 10 runs of NMF, both vanilla NMF and locaNMF, the robustness of the results can be seen from all of the 10 runs, where each run has some random effect. I guess the reasonable picture will be that locaNMF is very robust for all of the 10 runs, but vanilla NMF will not be the case. To summarize, could the authors give a few more demonstrations where the outcomes of different runs for vanilla NMF and locaNMF can be shown? Guess the runs should be like vanilla NMF + random initiation, vanilla NMF + brain atlas initiation, and locaNMF. 
The robustness is an important factor and LocaNMF provides a clear advantage here. We thank the reviewer for suggesting this analysis. We have now included Figure SI 3 showing the similarity between the components across different initializations of vanilla NMF, as compared to that using LocaNMF, on an example session. As we can see, LocaNMF provides a significant advantage in robustness over vanilla NMF with random initialization. While vanilla NMF with an atlas initialization is also robust across different initializations on the same dataset, it does not provide localized components, leading to a lack of consistency when comparing components across different sessions. This is now shown in Figure SI 6, and discussed in the Results section.

(2) When using locaNMF to WFCI data, both spatial and temporal components are important. In practice, the variation of experimental data is large. Dependent on experimental conditions, there are some cases where neurons in one part of the brain region could be temporally silent during some period. Maybe it will be useful to discuss this point a bit. With the 2 datasets used in this study, could we say something about the temporal dynamics? Or to characterize the activity degree a bit across different brain regions? It could be possible that WFCl is generally good enough that neurons are always active in some way. Maybe it is difficult to find a proper exp. data, then a simulated data, like the one used in this study, could help by changing their temporal dynamics a bit to generate some imbalanced states.

The activity in the different regions indeed has different amplitudes. We have now included Figure SI 7 showing the maximal variance of the temporal components in the different regions. Indeed, there is a considerable difference in variance across different regions (about an order of magnitude). This does not provide any problems for LocaNMF. For example, take the case where a region is temporally silent, but a nearby region is very active. Using vanilla NMF, the silent component may be merged into the active component while not being represented very well. However, using LocaNMF, this component would not have a high localization, and the corresponding lambda parameter would increase till the component is well represented.

(3) Similar to question (2), it seems that some data were not precisely aligned to the CCF, e.g. Fig 6 and 7. For example, the plot in Fig.6(D), if one sort the brain regions according to the similarity and plot after sorting. There are a few regions having a low index of similarity, for instance, RS part. Then for these less similar regions, what could be the reason? Because of their low active temporal dynamics? In another word, again, by simulated data, one can explore this in detail, I think. Then, one can get some guidelines that under which conditions, locaNMF may fail. These conditions may work together with the sensitivity of the hyperparameters. For example, different levels of localization threshold may depend on the temporal dynamics? While the hyperparameters per region inherently depend on the data, including the temporal dynamics, we perform a search over these hyperparameters to resolve this. In fact, while addressing this comment, we realized that we had been slightly underestimating the value for cosine similarity in our previous iteration of figures, and have thus updated the relevant figures to reflect this change. We apologize for this error, and we note that all relevant results and conclusions still hold. We are grateful to the reviewer's comment that allowed this to be brought to our attention. Indeed, there is still a variance in the index of similarity for different regions. 
However, the low index of similarity for some regions may indeed be due to the inherent difference in the spatial components between different sessions.

(4) line 573: "For runtime of LocaNMF on datasets of several sizes, see the Results section." It seems that there is no such part of the results in the text.

We had included the median runtime in the main text of the Results (lines 151-153); LocaNMF took a median of 29 minutes per session (on recordings of median length 1 hour).

(5) Line 258k dPCs is not defined.

Fixed; thanks.

(6) Line 548 sNMF is not defined.

Fixed; thanks again. 